\title{
An Accurate Block Hybrid Collocation Method for Third Order Ordinary Differential Equations
}

\author{
Lee Ken Yap, ${ }^{1,2}$ Fudziah Ismail, ${ }^{2}$ and Norazak Senu ${ }^{2}$ \\ ${ }^{1}$ Department of Mathematical and Actuarial Sciences, Universiti Tunku Abdul Rahman, Setapak, 53300 Kuala Lumpur, Malaysia \\ ${ }^{2}$ Department of Mathematics and Institute for Mathematical Research, Universiti Putra Malaysia, 43400 Serdang, Selangor, Malaysia \\ Correspondence should be addressed to Lee Ken Yap; lkyap@utar.edu.my
}

Received 24 January 2014; Revised 24 April 2014; Accepted 7 May 2014; Published 27 May 2014

Academic Editor: Kai Diethelm

Copyright (c) 2014 Lee Ken Yap et al. This is an open access article distributed under the Creative Commons Attribution License, which permits unrestricted use, distribution, and reproduction in any medium, provided the original work is properly cited.

\begin{abstract}
The block hybrid collocation method with two off-step points is proposed for the direct solution of general third order ordinary differential equations. Both the main and additional methods are derived via interpolation and collocation of the basic polynomial. These methods are applied in block form to provide the approximation at five points concurrently. The stability properties of the block method are investigated. Some numerical examples are tested to illustrate the efficiency of the method. The block hybrid collocation method is also implemented to solve the nonlinear Genesio equation and the problem in thin film flow.
\end{abstract}

\section{Introduction}

Consider the general third order ordinary differential equations (ODEs):

$$
y^{\prime \prime \prime}=f\left(x, y, y^{\prime}, y^{\prime \prime}\right),
$$

with the initial conditions

$$
y(a)=y_{0}, \quad y^{\prime}(a)=y_{0}^{\prime}, \quad y^{\prime \prime}(a)=y_{0}^{\prime \prime}, \quad x \in[a, b] .
$$

In particular, the third order differential equations arise in many physical problems such as electromagnetic waves, thin film flow, and gravity-driven flows (see [1-6]). Therefore, third order ODEs have attracted considerable attention. Many theoretical and numerical studies dealing with such equations have appeared in the literature. The popular approach for solving third order ODEs is by converting the problems to a system of first order ODEs and solving it using the method available in the literature. Awoyemi and Idowu [7], Jator [8], Mehrkanoon [9], and Bhrawy and Abd-Elhameed [10] remarked the drawback of this approach whereby it required complicated computational work and lengthy execution time. The studies on direct approach to higher order ODEs demonstrated the advantages in speed and accuracy.
Some attentions $[8,11-14]$ have been focused on direct solution of second order ODEs. Fatunla [12] suggested the zero-stable 2-point block method to solve special second order ODEs. On the other hand, Omar et al. [13] and Majid and Suleiman [14] studied parallel implementation of the direct block methods. Jator $[8,11]$ proposed a class of hybrid collocation methods and emphasized the accuracy advantage on self-starting method. The only necessary starting value for evaluation at the next block is the last value from the previous block. Since the loss of accuracy does not affect the subsequent points, the order of the method is maintained.

Some attempts have been made to solve third order ODEs directly using collocation method. Awoyemi [15] considered the P-stable linear multistep collocation method. Meanwhile, Awoyemi and Idowu [7] proposed the hybrid collocation method with an off-step point, $x_{n+3 / 2}$. Both schemes are implemented in predictor-corrector mode to obtain the approximation at $x_{n+3}$. The Taylor series expansion is employed for the computation of initial values. Olabode and Yusuph [16] applied the interpolation and collocation technique on power series to derive 3-step block method, and it was implemented as simultaneous integrator to special third order ODEs. Bhrawy and Abd-Elhameed [10] developed the shifted Jacobi-Gauss collocation spectral method for general nonlinear third order differential equations. 
Adesanya et al. [17] proposed a self-starting block predictorcorrector method whereby the derivation involved interpolation and collocation of power series at $x_{n+j}$, for $j=1(1) 3$ and $j=0(1) 4$, respectively.

Several direct variable step methods have also been proposed in literature to solve general third order ODEs. For instance, Mehrkanoon [9] implemented the direct threepoint block multistep method of Adams type formulas in PECE mode with variable step size and Gauss Seidel iteration. Majid et al. [18] presented the 2-point 4-step implicit block method with the application of the simple form in AdamsMoulton method using variable step size.

Here, we are going to derive the block hybrid collocation method for the direct solution of general third order ODEs. The method is along the lines proposed by Jator [11] and Awoyemi and Idowu [7]. The derivation involves interpolation and collocation of the basic polynomial. The collocation method approximates the solution of $y$ with basic polynomial which satisfies the initial conditions and differential equations at all points. This approach generates the main and the additional methods which can be combined and used as block method. In $m$-point block method, the interval is divided into series of blocks with each block containing $m$-points. The application of $m$-point block method generates a block of new solution concurrently.

\section{Derivation of Block Hybrid Collocation Methods}

The hybrid collocation method that produces approximations $y_{n+k}, y_{n+k}^{\prime}$, and $y_{n+k}^{\prime \prime}$ to the general third order ODEs is given as follows:

$$
\sum_{j=0}^{k} \alpha_{j} y_{n+j}+\sum_{j=1}^{2} \alpha_{v_{j}} y_{n+v_{j}}=h^{3}\left(\sum_{j=0}^{k} \beta_{j} f_{n+j}+\sum_{j=1}^{2} \beta_{v_{j}} f_{n+v_{j}}\right) .
$$

In order to obtain (3), we approximate the solution by the interpolating function $Y(x)$ of the form

$$
Y(x)=\sum_{j=0}^{r+s-1} \phi_{j} x^{j}
$$

where

(i) $x \in[a, b]$,

(ii) $\phi_{j}$ are unknown coefficients to be determined,

(iii) $r$ is the number of interpolations for $1 \leq r \leq k$, and

(iv) $s$ is the number of distinct collocation points with $s>$ 0 .

The continuous approximation is constructed by imposing the following conditions:

$$
\begin{gathered}
Y\left(x_{n+j}\right)=y_{n+j}, \quad j=0,1,2, \ldots, r-1, \\
Y^{\prime \prime \prime}\left(x_{n+\mu}\right)=f_{n+\mu}, \quad \mu=\left\{j, v_{1}, v_{2}\right\}, j=0,1,2, \ldots, k,
\end{gathered}
$$

where $v_{1}$ and $v_{2}$ are not integers. Interpolating (5) at the points $x_{n}, x_{n+1}, x_{n+2}$ and collocating (6) at the points $x_{n}$, $x_{n+1 / 2}, x_{n+1}, x_{n+3 / 2}, x_{n+2}$, and $x_{n+3}$ lead to a system of nine equations, which can be solved by Mathematica software to obtain the coefficient $\phi_{j}$. The values of $\phi_{j}$ are substituted into (4) to obtain the continuous multistep method of the form

$$
Y(x)=\sum_{j=0}^{k} \alpha_{j} y_{n+j}+h^{3}\left(\sum_{j=0}^{k} \beta_{j} f_{n+j}+\sum_{j=1}^{2} \beta_{v_{j}} f_{n+v_{j}}\right),
$$

where $\alpha_{j}, \beta_{j}$, and $\beta_{v_{j}}$ are constant coefficients. Hence, the block hybrid collocation method can be derived as follows.

Main Method. Consider

$$
\begin{aligned}
y_{n+3}= & 3 y_{n+2}-3 y_{n+1}+y_{n}+\frac{h^{3}}{90} \\
& \times\left(f_{n}+36 f_{n+1}+16 f_{n+3 / 2}+36 f_{n+2}+f_{n+3}\right) .
\end{aligned}
$$

Additional Method. Consider

$$
\begin{aligned}
y_{n+3 / 2}= & \frac{3}{8} y_{n+2}+\frac{3}{4} y_{n+1}-\frac{1}{8} y_{n}+\frac{h^{3}}{92160} \\
& \times\left(-19 f_{n}-648 f_{n+1 / 2}-2979 f_{n+1}\right. \\
& \left.-2104 f_{n+3 / 2}-9 f_{n+2}-f_{n+3}\right), \\
y_{n+1 / 2}= & -\frac{1}{8} y_{n+2}+\frac{3}{4} y_{n+1}+\frac{3}{8} y_{n}+\frac{h^{3}}{92160} \\
& \times\left(29 f_{n}+2040 f_{n+1 / 2}+3069 f_{n+1}\right. \\
& \left.+584 f_{n+3 / 2}+39 f_{n+2}-f_{n+3}\right) .
\end{aligned}
$$

It is noted that the general third order ODEs involve the first and second derivatives. These derivatives can be obtained by imposing that

$$
\begin{gathered}
Y^{\prime}(x)=\frac{1}{h}\left(\sum_{j=0}^{k} \alpha_{j}^{\prime} y_{n+j}+h^{3}\left(\sum_{j=0}^{k} \beta_{j}^{\prime} f_{n+j}+\sum_{j=1}^{2} \beta_{v_{j}}^{\prime} f_{n+v_{j}}\right)\right) \\
Y^{\prime \prime}(x)=\frac{1}{h^{2}}\left(\sum_{j=0}^{k} \alpha_{j}^{\prime \prime} y_{n+j}\right. \\
\left.+h^{3}\left(\sum_{j=0}^{k} \beta_{j}^{\prime \prime} f_{n+j}+\sum_{j=1}^{2} \beta_{v_{j}}^{\prime \prime} f_{n+v_{j}}\right)\right) .
\end{gathered}
$$

The values of $\phi_{j}$ are substituted into

$$
Y^{\prime}(x)=\sum_{j=1}^{r+s-1} j \phi_{j} x^{j-1}, \quad Y^{\prime \prime}(x)=\sum_{j=2}^{r+s-1} j(j-1) \phi_{j} x^{j-2}
$$


to generate the formula for the first and second derivatives of the method. Thus, we obtain

$$
\begin{aligned}
& h y_{n}^{\prime}=-\frac{1}{2} y_{n+2}+2 y_{n+1}-\frac{3}{2} y_{n}+\frac{h^{3}}{7560} \\
& \times\left(118 f_{n}+1296 f_{n+1 / 2}+819 f_{n+1}\right. \\
& \left.+304 f_{n+3 / 2}-18 f_{n+2}+f_{n+3}\right), \\
& h y_{n+1 / 2}^{\prime}=y_{n+1}-y_{n}+\frac{h^{3}}{241920} \\
& \times\left(-449 f_{n}-9312 f_{n+1 / 2}-99 f_{n+1}\right. \\
& \left.-320 f_{n+3 / 2}+105 f_{n+2}-5 f_{n+3}\right), \\
& h y_{n+1}^{\prime}=\frac{1}{2} y_{n+2}-\frac{1}{2} y_{n}+\frac{h^{3}}{2520} \\
& \times\left(-f_{n}-80 f_{n+1 / 2}-258 f_{n+1}\right. \\
& \left.-80 f_{n+3 / 2}-f_{n+2}\right) \text {, } \\
& h y_{n+3 / 2}^{\prime}=y_{n+2}-y_{n+1}+\frac{h^{3}}{241920} \\
& \times\left(5 f_{n}-549 f_{n+1}-8992 f_{n+3 / 2}\right. \\
& \left.-549 f_{n+2}+5 f_{n+3}\right), \\
& h y_{n+2}^{\prime}=\frac{3}{2} y_{n+2}-2 y_{n+1}+\frac{1}{2} y_{n}+\frac{h^{3}}{7560} \\
& \times\left(2 f_{n}+240 f_{n+1 / 2}+909 f_{n+1}\right. \\
& \left.+1232 f_{n+3 / 2}+138 f_{n+2}-f_{n+3}\right), \\
& h y_{n+3}^{\prime}=\frac{5}{2} y_{n+2}-4 y_{n+1}+\frac{3}{2} y_{n}+\frac{h^{3}}{7560} \\
& \times\left(343 f_{n}-1296 f_{n+1 / 2}+7758 f_{n+1}\right. \\
& \left.-2000 f_{n+3 / 2}+8595 f_{n+2}+460 f_{n+3}\right) \text {, } \\
& h^{2} y_{n}^{\prime \prime}=y_{n+2}-2 y_{n+1}+y_{n}+\frac{h^{3}}{2520} \\
& \times\left(-398 f_{n}-1584 f_{n+1 / 2}-201 f_{n+1}\right. \\
& \left.-400 f_{n+3 / 2}+66 f_{n+2}-3 f_{n+3}\right) \text {, } \\
& h^{2} y_{n+1 / 2}^{\prime \prime}=y_{n+2}-2 y_{n+1}+y_{n}+\frac{h^{3}}{40320} \\
& \times\left(345 f_{n}-5744 f_{n+1 / 2}-13443 f_{n+1}\right. \\
& \left.-912 f_{n+3 / 2}-421 f_{n+2}+15 f_{n+3}\right), \\
& h^{2} y_{n+1}^{\prime \prime}=y_{n+2}-2 y_{n+1}+y_{n}+\frac{h^{3}}{7560} \\
& \times\left(-11 f_{n}+624 f_{n+1 / 2}+90 f_{n+1}\right. \\
& \left.-752 f_{n+3 / 2}+51 f_{n+2}-2 f_{n+3}\right) \text {, }
\end{aligned}
$$

$$
\begin{aligned}
h^{2} y_{n+3 / 2}^{\prime \prime}= & y_{n+2}-2 y_{n+1}+y_{n}+\frac{h^{3}}{40320} \\
& \times\left(121 f_{n}+1872 f_{n+1 / 2}+12093 f_{n+1}\right. \\
& \left.+6704 f_{n+3 / 2}-645 f_{n+2}+15 f_{n+3}\right), \\
h^{2} y_{n+2}^{\prime \prime}= & y_{n+2}-2 y_{n+1}+y_{n}+\frac{h^{3}}{2520} \\
& \times\left(-6 f_{n}+208 f_{n+1 / 2}+471 f_{n+1}\right. \\
& \left.+1392 f_{n+3 / 2}+458 f_{n+2}-3 f_{n+3}\right), \\
h^{2} y_{n+3}^{\prime \prime}= & y_{n+2}-2 y_{n+1}+y_{n}+\frac{h^{3}}{2520} \\
& \times\left(295 f_{n}-1584 f_{n+1 / 2}+4902 f_{n+1}\right. \\
& \left.\quad-4432 f_{n+3 / 2}+5169 f_{n+2}+690 f_{n+3}\right) .
\end{aligned}
$$

\section{Order and Stability Properties}

Extending the idea of Henrici [19] and Jator [8], the linear difference operator $L$ associated with (3) is defined by

$$
\begin{aligned}
L[y(x) ; h]= & \sum_{j=0}^{k}\left[\alpha_{j} y(x+j h)-h^{3} \beta_{j} y^{\prime \prime \prime}(x+j h)\right] \\
& +\sum_{j=1}^{2}\left[\alpha_{v_{j}} y\left(x+v_{j} h\right)-h^{3} \beta_{v_{j}} y^{\prime \prime \prime}\left(x+v_{j} h\right)\right],
\end{aligned}
$$

where $y(x)$ is an arbitrary function that is sufficiently differentiable. We expand the test function $y(x+j h)$ and its third derivative $y^{\prime \prime \prime}(x+j h)$ about $x$ and collect the terms to obtain

$$
L[y(x) ; h]=C_{0} y(x)+C_{1} h y^{\prime}(x)+\cdots+C_{q} h^{q} y^{(q)}(x)+\cdots
$$

whose coefficients $C_{q}$ for $q=0,1, \ldots$ are constants and given as

$$
\begin{aligned}
C_{0}= & \sum_{j=0}^{k} \alpha_{j}+\sum_{j=1}^{2} \alpha_{v_{j}} \\
C_{1}= & \sum_{j=1}^{k} j \alpha_{j}+\sum_{j=1}^{2} v_{j} \alpha_{v_{j}} \\
\vdots & \\
C_{q}= & \frac{1}{q !}\left[\sum_{j=1}^{k} j^{q} \alpha_{j}+\sum_{j=1}^{2} v_{j}^{q} \alpha_{v_{j}}\right] \\
& -\frac{1}{(q-3) !}\left[\sum_{j=1}^{k} j^{q-3} \beta_{j}+\sum_{j=1}^{2} v_{j}^{q-3} \beta_{v_{j}}\right] .
\end{aligned}
$$


The associated linear multistep method and the linear difference operator are said to be of order $p$, if $C_{0}=C_{1}=$ $\cdots=C_{p+2}=0$ and $C_{p+3} \neq 0$. The main method and the two additional methods have order $p=6$ with the error constants; $C_{9}$ are $-19 / 120960,41 / 61931520$, and $41 / 61931520$, respectively. The block hybrid collocation method is consistent since it has order $p>1$.

To analyze the zero stability, (8)-(12) are normalized. Zero stability can be described by matrix finite difference equation as follows:

$$
\begin{aligned}
I_{5} Y_{m+1}= & A^{(1)} Y_{m}+h^{3}\left(B^{(0)} F_{m+1}+B^{(1)} F_{m}\right) \\
& +h^{2} C^{(1)} Y_{m}^{\prime \prime}+h D^{(1)} Y_{m}^{\prime}
\end{aligned}
$$

with

$$
\begin{aligned}
Y_{m+1} & =\left[y_{n+1 / 2}, y_{n+1}, y_{n+3 / 2}, y_{n+2}, y_{n+3}\right]^{T} \\
Y_{m} & =\left[y_{n-2}, y_{n-3 / 2}, y_{n-1}, y_{n-1 / 2}, y_{n}\right]^{T} \\
F_{m+1} & =\left[f_{n+1 / 2}, f_{n+1}, f_{n+3 / 2}, f_{n+2}, f_{n+3}\right]^{T} \\
F_{m} & =\left[f_{n-2}, f_{n-3 / 2}, f_{n-1}, f_{n-1 / 2}, f_{n}\right]^{T}, \\
Y_{m}^{\prime \prime} & =\left[y_{n-2}^{\prime \prime}, y_{n-3 / 2}^{\prime \prime}, y_{n-1}^{\prime \prime}, y_{n-1 / 2}^{\prime \prime}, y_{n}^{\prime \prime}\right]^{T} \\
Y_{m}^{\prime} & =\left[y_{n-2}^{\prime}, y_{n-3 / 2}^{\prime}, y_{n-1}^{\prime}, y_{n-1 / 2}^{\prime}, y_{n}^{\prime}\right]^{T}
\end{aligned}
$$

and constant matrices

$$
\begin{gathered}
I_{5}=5 \times 5 \text { identity matrix; } \\
A^{(1)}=\left[\begin{array}{ccccc}
0 & -\frac{9}{8} & 0 & -\frac{27}{16} & \frac{3}{8} \\
0 & \frac{9}{2} & 0 & \frac{27}{4} & -\frac{3}{2} \\
0 & \frac{3}{8} & 0 & \frac{9}{16} & -\frac{1}{8} \\
0 & -3 & 0 & -\frac{9}{2} & 1 \\
0 & -3 & 0 & -\frac{9}{2} & 1
\end{array}\right] ; \\
B^{(0)}=\left[\begin{array}{ccccc}
\frac{7}{768} & -\frac{7}{128} & \frac{73}{11520} & -\frac{7}{256} & -\frac{1}{92160} \\
\frac{6}{35} & -\frac{1}{4} & \frac{38}{945} & -\frac{7}{60} & \frac{1}{7560} \\
-\frac{9}{1280} & \frac{9}{128} & -\frac{263}{11520} & \frac{11}{256} & -\frac{1}{92160} \\
-\frac{22}{35} & \frac{7}{12} & -\frac{10}{63} & \frac{1}{4} & -\frac{1}{840} \\
0 & -\frac{3}{2} & \frac{8}{45} & -\frac{5}{4} & \frac{1}{90}
\end{array}\right] ;
\end{gathered}
$$

$$
\begin{aligned}
& B^{(1)}=\left[\begin{array}{ccccc}
0 & -\frac{29}{30720} & 0 & -\frac{29}{20480} & \frac{29}{92160} \\
0 & -\frac{59}{1260} & 0 & -\frac{59}{840} & \frac{59}{3780} \\
0 & \frac{19}{30720} & 0 & \frac{19}{20480} & -\frac{19}{92160} \\
0 & \frac{199}{420} & 0 & \frac{199}{280} & -\frac{199}{1260} \\
0 & -\frac{1}{30} & 0 & -\frac{1}{20} & \frac{1}{90}
\end{array}\right] \\
& C^{(1)}=\left[\begin{array}{ccccc}
0 & 0 & 0 & 0 & 0 \\
0 & 0 & 0 & 0 & 0 \\
0 & 0 & 0 & 0 & 0 \\
& & & & \\
0 & 3 & 0 & \frac{9}{2} & -1 \\
0 & 0 & 0 & 0 & 0
\end{array}\right] ; \quad D^{(1)}=\left[\begin{array}{ccccc}
0 & 0 & 0 & 0 & 0 \\
& & & & \\
0 & 3 & 0 & \frac{9}{2} & -1 \\
0 & 0 & 0 & 0 & 0 \\
0 & 0 & 0 & 0 & 0 \\
0 & 0 & 0 & 0 & 0
\end{array}\right] .
\end{aligned}
$$

The first characteristic polynomial is defined as

$$
\rho(z)=\operatorname{Det}\left[z A^{(0)}-A^{(1)}\right]=z^{4}(z-1) .
$$

Since the roots of the first characteristic polynomial are modulus less than or equal to one, the method is zero stable. This property, together with the consistency of the method, implies the convergence (see Henrici [19]) of the block method.

\section{Numerical Examples and Discussion}

To illustrate the effectiveness of the block hybrid collocation method, the test Problems 1-3 are solved numerically. The block hybrid collocation method is implemented and leads to a system of fifteen equations. The code is written and executed in Mathematica 8.0 to obtain the numerical solutions. A built-in Mathematica package, namely, LinearSolve $[m, b]$, is applied in the algorithm to solve the linear system for numerical results. In general, this package solves the matrix equation $m \cdot x=b$ and returns a vector $x$.

The block hybrid collocation method is then compared with the existing methods [7, 15-17, 20] for direct solution of general third order ODEs. The block hybrid collocation method is also compared with the Adams Bashforth-Adams Moulton method. The well-known fourth order Runge Kutta method is applied to obtain the starting values for Adams method, whereby the third order ODEs are reduced to system of first order ODEs.

Problem 1. Consider

$$
\begin{aligned}
& y^{\prime \prime \prime}-2 y^{\prime \prime}-3 y^{\prime}+10 y= 34 x e^{-2 x}-16 e^{-2 x} \\
&-10 x^{2}+6 x+34 \\
& y(0)=3, \quad y^{\prime}(0)=y^{\prime \prime}(0)=0, \quad x \in[0, b] .
\end{aligned}
$$

Exact Solution: $y(x)=x^{2} e^{-2 x}-x^{2}+3$.

Source: Majid et al. [18]. 
TABLE 1: Numerical results for Problem 1.

\begin{tabular}{|c|c|c|c|c|}
\hline$b$ & $h$ & Method & Step & Maxe \\
\hline \multirow{6}{*}{1.0} & \multirow{3}{*}{0.0125} & BHCM & 27 & $4.23 \times 10^{-12}$ \\
\hline & & Adams & 80 & $3.56 \times 10^{-8}$ \\
\hline & & Awoyemi(2) & 80 & $7.60 \times 10^{-6}$ \\
\hline & \multirow{3}{*}{0.00625} & BHCM & 54 & $7.24 \times 10^{-14}$ \\
\hline & & Adams & 160 & $3.23 \times 10^{-9}$ \\
\hline & & Awoyemi(2) & 160 & $9.54 \times 10^{-7}$ \\
\hline \multirow{6}{*}{4.0} & \multirow{3}{*}{0.01} & BHCM & 134 & $3.50 \times 10^{-11}$ \\
\hline & & Adams & 400 & $2.91 \times 10^{-6}$ \\
\hline & & Awoyemi(1) & 400 & $1.16 \times 10^{-3}$ \\
\hline & \multirow{3}{*}{0.005} & BHCM & 267 & $3.30 \times 10^{-14}$ \\
\hline & & Adams & 800 & $2.10 \times 10^{-7}$ \\
\hline & & Awoyemi(1) & 800 & $1.46 \times 10^{-4}$ \\
\hline
\end{tabular}

Problem 2. Consider

$$
\begin{gathered}
y^{\prime \prime \prime}+y^{\prime}=0 \\
y(0)=0, \quad y^{\prime}(0)=1, \quad y^{\prime \prime}(0)=2, \quad x \in[0, b] .
\end{gathered}
$$

Exact Solution: $y(x)=2(1-\cos x)+\sin x$.

Source: Majid et al. [18].

Problem 3. Consider

$$
\begin{gathered}
y^{\prime \prime \prime}=3 \sin x \\
y(0)=1, \quad y^{\prime}(0)=0, \quad y^{\prime \prime}(0)=-2, \quad x \in[0, b] .
\end{gathered}
$$

Exact Solution: $y(x)=3 \cos x+x^{2} / 2-2$.

Source: Adesanya et al. [17].

The performance comparison between block hybrid collocation method with the existing results $[7,9,15-17,20]$ and the Adams Bashforth-Adams Moulton method is presented in Tables 1-4. The following notations are used in the tables.

$h$ : step size.

BHCM: block hybrid collocation method.

Adams: Adams Bashforth-Adams Moulton method.

Awoyemi (1): P-stable multistep method in Awoyemi [15].

Awoyemi (2): hybrid collocation method in Awoyemi and Idowu [7].

Awoyemi (3): nonsymmetric collocation method in Awoyemi et al. [20].

Adesanya: block predictor-corrector method in Adesanya et al. [17].

Mehrkanoon: variable step three-point block multistep method in Mehrkanoon [9].

Olabode: block method for special third order ODEs in Olabode and Yusuph [16].

Step: total number of steps taken to obtain solution.
TABLe 2: Numerical results for Problem 2.

\begin{tabular}{lcccc}
\hline$b$ & $h$ & Method & Step & Maxe \\
\hline \multirow{4}{*}{1.0} & BHCM & 4 & $1.11 \times 10^{-10}$ \\
& 0.1 & Adams & 10 & $2.76 \times 10^{-6}$ \\
& & Awoyemi(3) & 10 & $1.07 \times 10^{-6}$ \\
& & Adesanya & 3 & $5.14 \times 10^{-5}$ \\
5.0 & BHCM & 67 & $1.19 \times 10^{-12}$ \\
& & Adams & 200 & $9.72 \times 10^{-8}$ \\
& & Awoyemi(1) & 200 & $3.53 \times 10^{-6}$ \\
10.0 & BHCM & 134 & $2.11 \times 10^{-12}$ \\
& & Adams & 400 & $1.83 \times 10^{-7}$ \\
& & Awoyemi(1) & 400 & $2.25 \times 10^{-6}$ \\
15.0 & & BHCM & 200 & $2.33 \times 10^{-12}$ \\
& & Adams & 600 & $3.16 \times 10^{-7}$ \\
& & Awoyemi(1) & 600 & $9.85 \times 10^{-6}$ \\
20.0 & BHCM & 267 & $3.48 \times 10^{-12}$ \\
& & Adams & 800 & $4.58 \times 10^{-7}$ \\
& & Awoyemi(1) & 800 & $6.31 \times 10^{-6}$ \\
\hline
\end{tabular}

TAble 3: Numerical results for Problem 3.

\begin{tabular}{ccccc}
\hline$b$ & $h$ & Method & Step & Maxe \\
\hline \multirow{4}{*}{1.0} & \multirow{4}{*}{0.01} & BHCM & 34 & $7.48 \times 10^{-17}$ \\
& & Adams & 100 & $6.40 \times 10^{-10}$ \\
& & Olabode & 34 & $8.89 \times 10^{-13}$ \\
& & Adesanya & 25 & $1.75 \times 10^{-14}$ \\
\hline
\end{tabular}

TABLE 4: Comparison of numerical results for nonlinear Genesio equation.

\begin{tabular}{ccccccc}
\hline \multirow{2}{*}{$b$} & \multicolumn{3}{c}{ Mehrkanoon } & \multicolumn{3}{c}{ BHCM } \\
& TOL & Step & $y$ & $h$ & Step & $y$ \\
\hline \multirow{2}{*}{1.0} & $10^{-6}$ & 17 & -0.054005 & 0.1 & 4 & -0.05400408324564678 \\
& $10^{-10}$ & 26 & -0.054003 & 0.01 & 34 & -0.05400408355473926 \\
\hline \multirow{2}{*}{4.0} & $10^{-6}$ & 23 & -0.067921 & 0.1 & 13 & -0.06763059062408930 \\
& $10^{-10}$ & 45 & -0.067692 & 0.01 & 133 & -0.06763060515900272 \\
\hline
\end{tabular}

Time: execution time taken in microseconds.

Maxe: magnitude of the maximum error of the computed solution.

The maximum error is defined as

$$
\text { Maxe }=\max _{1 \leq i \leq \text { Step }}\left(\left|y\left(x_{i}\right)-y_{i}\right|\right) .
$$

Tables 1-3 show that the block hybrid collocation method has better performance in terms of accuracy and number of steps to obtain the solution compared to Adams BashforthAdams Moulton method.

In Table 1, a direct comparison is made between Awoyemi methods [7, 15] and our block hybrid collocation method. For $b=1$, BHCM has better approximation compared to Awoyemi hybrid collocation method [7] for step sizes $h=$ 0.0125 and $h=0.00625$. Even in the larger interval $(b=$ 4), BHCM has higher order of accuracy when compared to Awoyemi P-stable multistep method [15] using $h=0.01$ and 


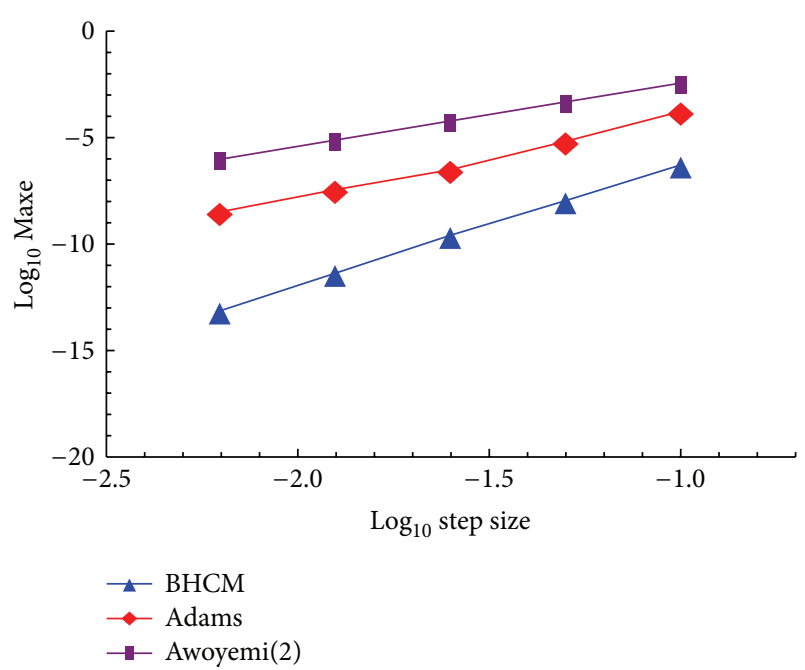

FIgURE 1: Performance comparison for Problem 1 when $b=1$.

$h=0.005$. It is obvious that BHCM requires less steps to obtain the solutions compared to Awoyemi methods $[7,15]$.

Table 2 shows that BHCM manages to achieve better accuracy and less total steps compared to Awoyemi P-stable multistep method [15] for constant step size 0.025 when $b=$ $5,10,15$, and 20. Furthermore, BHCM gain better accuracy compared to Awoyemi nonsymmetric collocation method [20] and Adesanya block predictor-corrector method [17] when $h=0.1$ and $b=1$. Table 3 shows the superiority of $\mathrm{BHCM}$ in terms of accuracy over Adesanya block predictorcorrector method [17] and Olabode block method [16] for special third order ODEs.

Figures 1,2, and 3 depict the performance comparison between BHCM and the existing methods: Awoyemi hybrid collocation method [7], Awoyemi P-stable multistep method [15], and Adams Bashforth-Adams Moulton method, respectively. It shows that BHCM is more efficient than the existing methods in terms of accuracy. BHCM also requires less computational time compared to the existing methods. This is expected since BHCM calculates the approximation at three main points concurrently.

\section{Application to Solve Nonlinear Genesio Equation}

Here we consider the nonlinear chaotic system from Genesio and Tesi [1]

$$
x^{\prime \prime \prime}+A x^{\prime \prime}+B x^{\prime}-f(x(t))=0
$$

with

$$
f(x(t))=-C x(t)+x^{2}(t)
$$

that is subject to the following initial conditions:

$$
\begin{array}{r}
x(0)=0.2, \quad x^{\prime}(0)=-0.3, \quad x^{\prime \prime}(0)=0.1, \\
t \in[0, b],
\end{array}
$$

where $A=1.2, B=2.92$, and $C=6$ are the positive constants that satisfied $A B<C$ for the existence of the solution.

BHCM is implemented together with a built-in Mathematica package, namely, FindRoot, for the solution of nonlinear system based on Newton's method. In order to apply BHCM and Newton's method at the next block, the last value from previous block is used as starting value and initial guess, respectively.

The numerical solutions are compared with the numerical solutions obtained in Mehrkanoon [9], Bataineh et al. [2], and the Mathematica built-in package NDSolve.

Table 4 shows the comparison in the numerical approximation of $y$ at the end points $b=1$ and $b=4$. BHCM achieves similar approximation as Mehrkanoon variable step three-point block multistep method [9].

Figure 4 depicts the numerical solutions for the nonlinear Genesio equation [1] in the interval $[0,4.5]$. Bataineh et al. [2] stated that NHAM is more stable than the numerical solution obtained by classical HAM. In fact, the solutions obtained by BHCM are in agreement with the observation of Bataineh et al. [2] using NHAM and Mathematica built-in package NDSolve.

\section{Application to Solve Problem in Thin Film Flow}

The proposed method is also applied to solve the well-known physical problem regarding the thin film flow of a liquid. In fluid dynamics, Tuck and Schwartz [3] investigated some third order ODEs that are relevant to draining and coating flows. They discussed the motion of the fluid on a plane surface in which the flow is in the direction of motion along the plane. This fluid dynamics problem was formulated in an autonomous third order ODEs

$$
y^{\prime \prime \prime}=f(y)
$$

where

$$
\begin{aligned}
& f(y)=-1+y^{-2}, \\
& f(y)=-1+\left(1+\delta+\delta^{2}\right) y^{-2}-\left(\delta+\delta^{2}\right) y^{-3}, \\
& f(y)=y^{-2}-y^{-3}, \\
& f(y)=y^{-2} .
\end{aligned}
$$

In the literature, some numerical methods for solving special third order ODEs have been extended to solve the problem in thin film flow. Some numerical investigation was presented in Momoniat and Mahomed [4] and Mechee et al. [5] concerning the special third order ODEs

$$
y^{\prime \prime \prime}=y^{-k}
$$

with the initial conditions $y(0)=y^{\prime}(0)=y^{\prime \prime}(0)=1$ for the cases $k=2$ and $k=3$.

Instead of using the conventional approach of reduction to system of first order ODEs, Momoniat and Mahomed [4] applied the successive reduction by writing the third order 


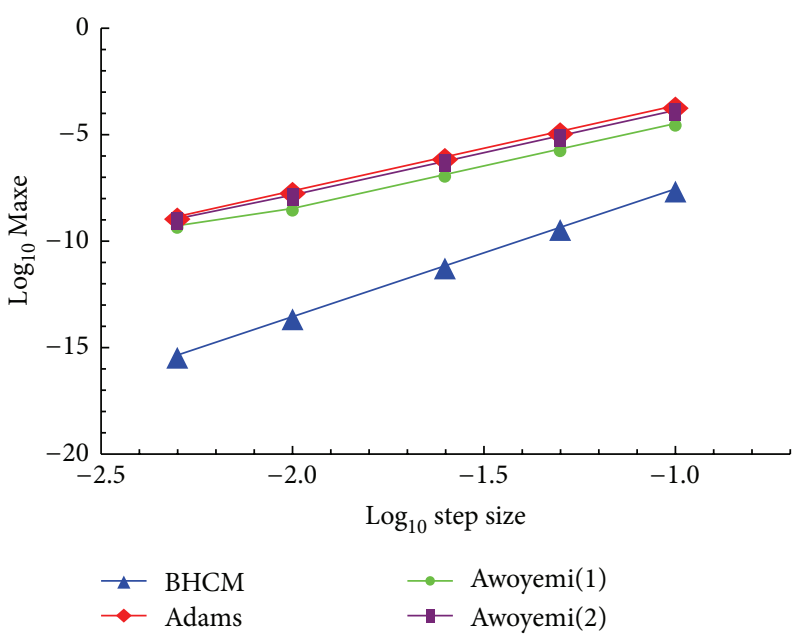

(a) Maxe versus step size

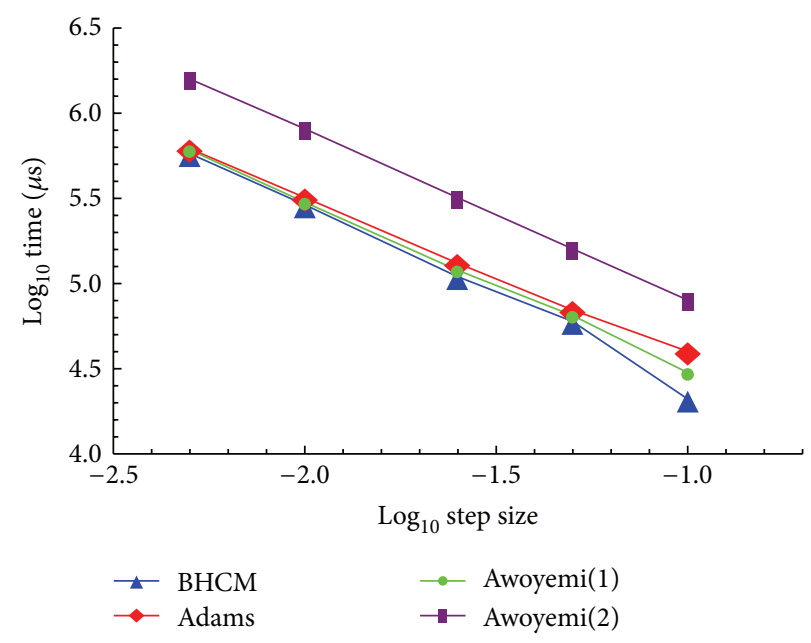

(b) Time versus step size

Figure 2: Performance comparison for Problem 2 when $b=40$.

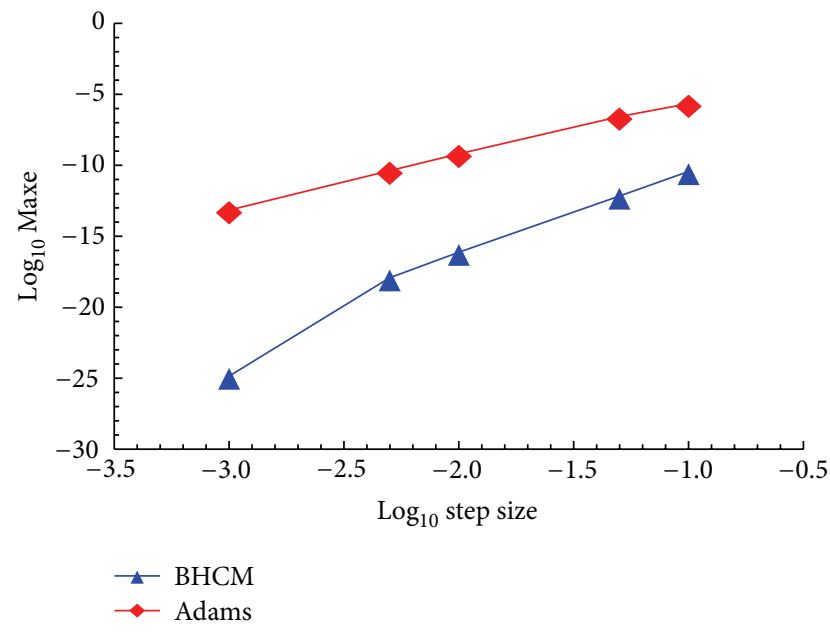

Figure 3: Performance comparison for Problem 3 when $b=1$.

ODEs (29) in terms of the differential invariants and solved it by fourth order Runge Kutta method. Mechee et al. [5] applied the three-stage fifth order Runge Kutta method to solve the third order physical problems (29) directly.

Here we will apply the proposed block hybrid collocation method (BHCM) to solve the third order ODEs (29). The numerical solutions are compared with Momoniat and Mahomed [4] and Mechee et al. [5] in the literature. The results are displayed in Tables 5-8.

Table 5 demonstrates that BHCM performs slightly better compared to Mechee et al. [5] with $h=0.1$ for the case $k=$ 2. In Table 6, we observe that Momoniat and Mahomed [4], Mechee et al. [5], and BHCM have similar order of accuracy.

Tables 7 and 8 show the numerical results for the case $k=3$ with $h=0.1$ and $h=0.01$, respectively. In fact, the case $k=3$ cannot be solved analytically. Table 7 shows that BHCM manages to achieve the numerical result which agrees to six decimal places when compared to Mechee et al. [5] for
TABLE 5: Numerical results for problem in Thin Film Flow (29) with $h=0.1, k=2$.

\begin{tabular}{lccc}
\hline$x$ & & Error \\
& Mechee & & BHCM \\
\hline 0.2 & $1.07 \times 10^{-6}$ & $1.03 \times 10^{-6}$ \\
0.4 & $4.13 \times 10^{-7}$ & $1.12 \times 10^{-7}$ \\
0.6 & $8.51 \times 10^{-7}$ & $2.07 \times 10^{-9}$ \\
0.8 & $1.71 \times 10^{-6}$ & $1.02 \times 10^{-8}$ \\
1.0 & $3.86 \times 10^{-6}$ & $9.35 \times 10^{-7}$ \\
\hline
\end{tabular}

TABLE 6: Numerical results for problem in Thin Film Flow (29) with $h=0.01, k=2$.

\begin{tabular}{lccc}
\hline$x$ & Momoniat & $\begin{array}{c}\text { Error } \\
\text { Mechee }\end{array}$ & BHCM \\
\hline 0.2 & $1.03 \times 10^{-6}$ & $1.03 \times 10^{-6}$ & $1.03 \times 10^{-6}$ \\
0.4 & $1.14 \times 10^{-7}$ & $1.13 \times 10^{-7}$ & $1.13 \times 10^{-7}$ \\
0.6 & $7.00 \times 10^{-9}$ & $6.30 \times 10^{-9}$ & $6.32 \times 10^{-9}$ \\
0.8 & $1.00 \times 10^{-9}$ & $1.00 \times 10^{-10}$ & $7.83 \times 10^{-11}$ \\
1.0 & $9.55 \times 10^{-7}$ & $9.54 \times 10^{-7}$ & $9.54 \times 10^{-7}$ \\
\hline
\end{tabular}

TABLE 7: Numerical results for problem in Thin Film Flow (29) with $h=0.1, k=3$.

\begin{tabular}{lcc}
\hline$x$ & Mechee & BHCM \\
\hline 0.0 & 1.0000000000 & 1.0000000000000000 \\
0.2 & 1.2211550887 & 1.2211551426800236 \\
0.4 & 1.4881049238 & 1.4881052873784077 \\
0.6 & 1.8042615558 & 1.8042625625912998 \\
0.8 & 2.1715208324 & 2.1715228333017014 \\
1.0 & 2.5909549758 & 2.5909583248983960 \\
\hline
\end{tabular}

$h=0.1$. In Table 8 , the numerical results for BHCM agree to nine decimal places when compared to Momoniat and 


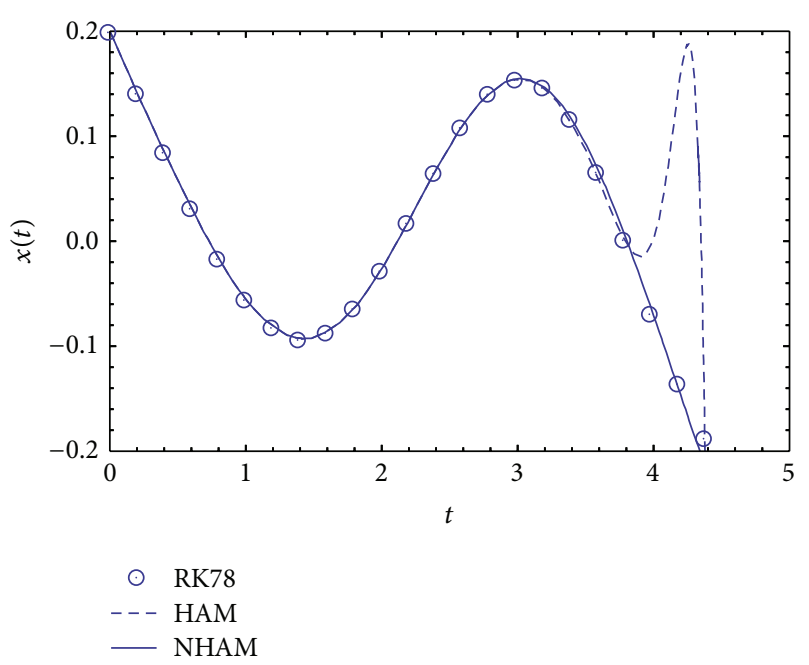

(a) Solutions obtained by seven- and eight-order Runge Kutta method (RK78), homotopy analysis method (HAM), and new variant of HAM (NHAM) (adapted from Bataineh et al. [2])

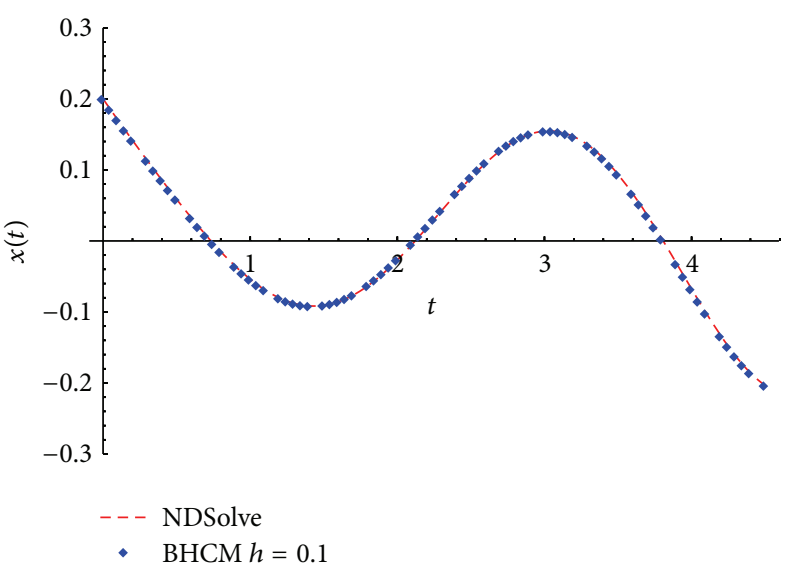

(b) Solutions obtained by BHCM with $h=0.1$

FIGURE 4: Plot of solutions for nonlinear Genesio equation.

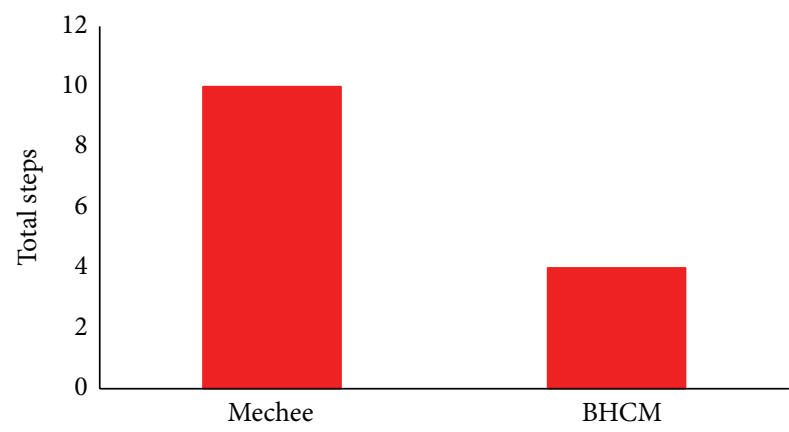

- $h=0.1$

(a) Total steps when $h=0.1$

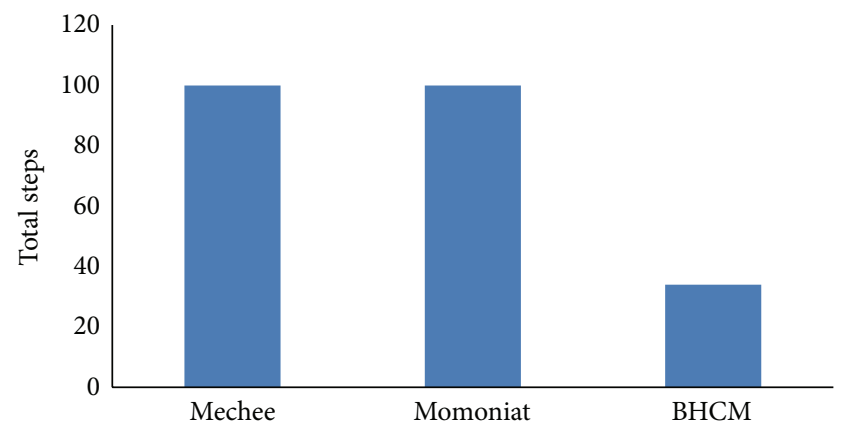

- $h=0.01$

FIGURE 5: Total steps VS method.

TABLe 8: Numerical results for problem in Thin Film Flow (29) with $h=0.01, k=3$.

\begin{tabular}{lccc}
\hline$x$ & Momoniat & Mechee & BHCM \\
\hline 0.0 & 1.000000000 & 1.0000000000 & 1.0000000000000000 \\
0.2 & 1.221155142 & 1.2211551423 & 1.2211551423957325 \\
0.4 & 1.488105284 & 1.4881052838 & 1.4881052842194118 \\
0.6 & 1.804262548 & 1.8042625471 & 1.8042625481474530 \\
0.8 & 2.171522797 & 2.1715227960 & 2.1715227981283490 \\
1.0 & 2.590958258 & 2.5909582556 & 2.5909582591167280
\end{tabular}

Mohamed [4], while they agree to ten decimal places when compared to Mechee et al. [5] for $h=0.01$.

Figure 5 shows the total number of steps taken to obtain the solutions versus method. It is obvious that BHCM require less steps to obtain the solution compared to Mechee et al. [5] and Momoniat and Mahomed [4]. The results are expected since the BHCM calculates the values of $y$ at 3 points simultaneously while the Runge Kutta method in Mechee et al. [5] and Momoniat and Mahomed [4] calculates only one value of $y$ at a time.

BHCM is clearly superior in solving the problem in thin film flow (29) since it involves less computational work and yields highly accurate solutions.

\section{Conclusion}

As a whole, the numerical results demonstrate the efficiency of the block hybrid collocation method. It is observed that the block hybrid collocation method requires less number of total steps compared to Awoyemi methods [7, 15, 20]. It reduces the total number of steps to almost one-third to obtain the solution. These results are expected since the 3point block methods calculate the values of $y$ at three main points concurrently. 
The numerical results also demonstrate the accuracy of the block hybrid collocation method. It gives precise approximation as the step size decreases. When compared with the existing methods $[7,15-17,20]$ and Adams Bashforth-Adams Moulton method, the block hybrid collocation method meets better accuracy for various step sizes even in larger interval.

As a conclusion, the 3-point block hybrid collocation method with two off-step points has been proposed and implemented as a self-starting method for third order ordinary differential equations. The results suggest a significant improvement in efficiency of the block hybrid collocation method in the direct solution of general third order ODEs. The block hybrid collocation method is applicable to solve the nonlinear Genesio equation [1] and the physical problem in thin film flow $[4,5]$.

\section{Conflict of Interests}

The authors declare that there is no conflict of interests regarding the publication of this paper.

\section{References}

[1] R. Genesio and A. Tesi, "Harmonic balance methods for the analysis of chaotic dynamics in nonlinear systems," Automatica, vol. 28, no. 3, pp. 531-548, 1992.

[2] A. S. Bataineh, M. S. M. Noorani, and I. Hashim, "Direct solution of $n$ th-order IVPs by homotopy analysis method," Differential Equations and Nonlinear Mechanics, vol. 2009, Article ID 842094, 15 pages, 2009.

[3] E. O. Tuck and L. W. Schwartz, "A numerical and asymptotic study of some third-order ordinary differential equations relevant to draining and coating flows," SIAM Review, vol. 32, no. 3, pp. 453-469, 1990.

[4] E. Momoniat and F. M. Mahomed, "Symmetry reduction and numerical solution of a third-order ODE from thin film flow," Mathematical and Computational Applications, vol. 15, no. 4, pp. 709-719, 2010.

[5] M. Mechee, N. Senu, F. Ismail, B. Nikouravan, and Z. Siri, "A three-stage fifth-order Runge-Kutta method for directly solving special third-order differential equation with application to thin film flow problem," Mathematical Problems in Engineering, vol. 2013, Article ID 795397, 7 pages, 2013.

[6] Y.J. L. Guo, "A third order equation arising in the falling film," Taiwanese Journal of Mathematics, vol. 11, no. 3, pp. 637-643, 2007.

[7] D. O. Awoyemi and O. M. Idowu, "A class of hybrid collocation methods for third-order ordinary differential equations," International Journal of Computer Mathematics, vol. 82, no. 10, pp. 1287-1293, 2005.

[8] S. N. Jator, "On a class of hybrid methods for $y^{\prime \prime}=f\left(x, y, y^{\prime}\right)$," International Journal of Pure and Applied Mathematics, vol. 59, no. 4, pp. 381-395, 2010.

[9] S. Mehrkanoon, "A direct variable step block multistep method for solving general third-order ODEs," Numerical Algorithms, vol. 57, no. 1, pp. 53-66, 2011.

[10] A. H. Bhrawy and W. M. Abd-Elhameed, "New algorithm for the numerical solutions of nonlinear third-order differential equations using Jacobi-Gauss collocation method," Mathematical Problems in Engineering, vol. 2011, Article ID 837218, 14 pages, 2011.
[11] S. N. Jator, "Solving second order initial value problems by a hybrid multistep method without predictors," Applied Mathematics and Computation, vol. 217, no. 8, pp. 4036-4046, 2010.

[12] S. O. Fatunla, "Block methods for second order ODEs," International Journal of Computer Mathematics, vol. 41, no. 1-2, pp. 55-63, 1991.

[13] Z. Omar, M. Sulaiman, M. Y. Saman, and D. J. Evans, "Parallel r-point explicit block method for solving second order ordinary differential equations directly," International Journal of Computer Mathematics, vol. 79, no. 3, pp. 289-298, 2002.

[14] Z. A. Majid and M. Suleiman, "Parallel direct integration variable step block method for solving large system of higher order ordinary differential equations," World Academy of Science, Engineeringand Technology, vol. 40, pp. 71-75, 2008.

[15] D. O. Awoyemi, "A P-stable linear multistep method for solving general third order ordinary differential equations," International Journal of Computer Mathematics, vol. 80, no. 8, pp. 985991, 2003.

[16] B. T. Olabode and Y. Yusuph, "A new block method for special third order ordinary differential equation," Journal of Mathematics and Statistics, vol. 5, no. 3, pp. 167-170, 2009.

[17] A. O. Adesanya, M. O. Udo, and A. M. Alkali, "A new block-predictorcorrector algorithm for the solution of $y^{\prime \prime \prime}=$ $f\left(x, y, y^{\prime \prime}\right)$," The American Journal of Computational Mathematics, vol. 2, no. 4, pp. 341-344, 2012.

[18] Z. A. Majid, N. A. Azmi, M. Suleiman, and Z. B. Ibrahim, "Solving directly general third order ordinary differential equations using two-point four step block method," Sains Malaysiana, vol. 41, no. 5, pp. 623-632, 2012.

[19] P. Henrici, Discrete Variable Methods in Ordinary Differential Equations, John Wiley \& Sons, New York, NY, USA, 1962.

[20] D. O. Awoyemi, M. O. Udoh, and A. O. Adesanya, "Nonsymmetric collocation method for direct solution of general third order initial value problems of ordinary differential equations," Journal of Natural and Applied Mathematics, vol. 7, pp. 31-37, 2006. 


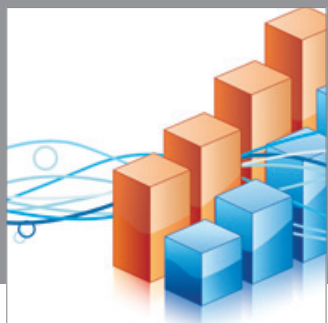

Advances in

Operations Research

mansans

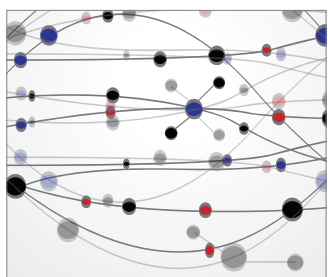

The Scientific World Journal
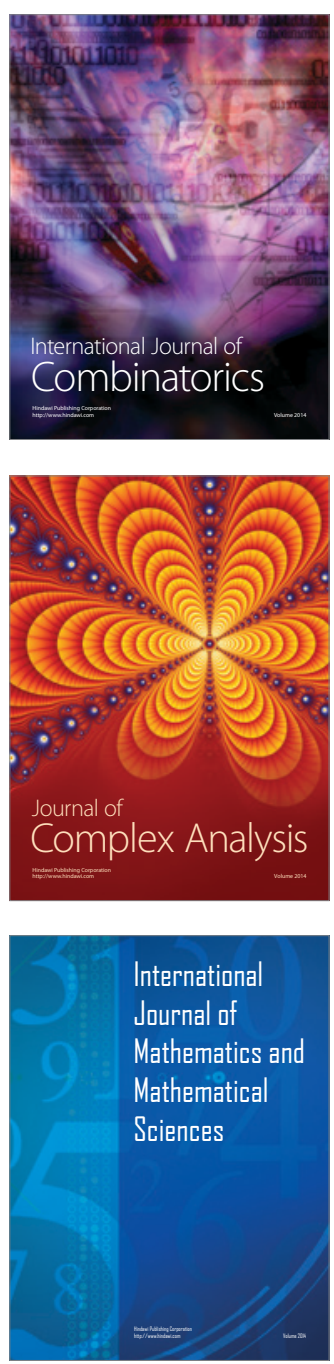
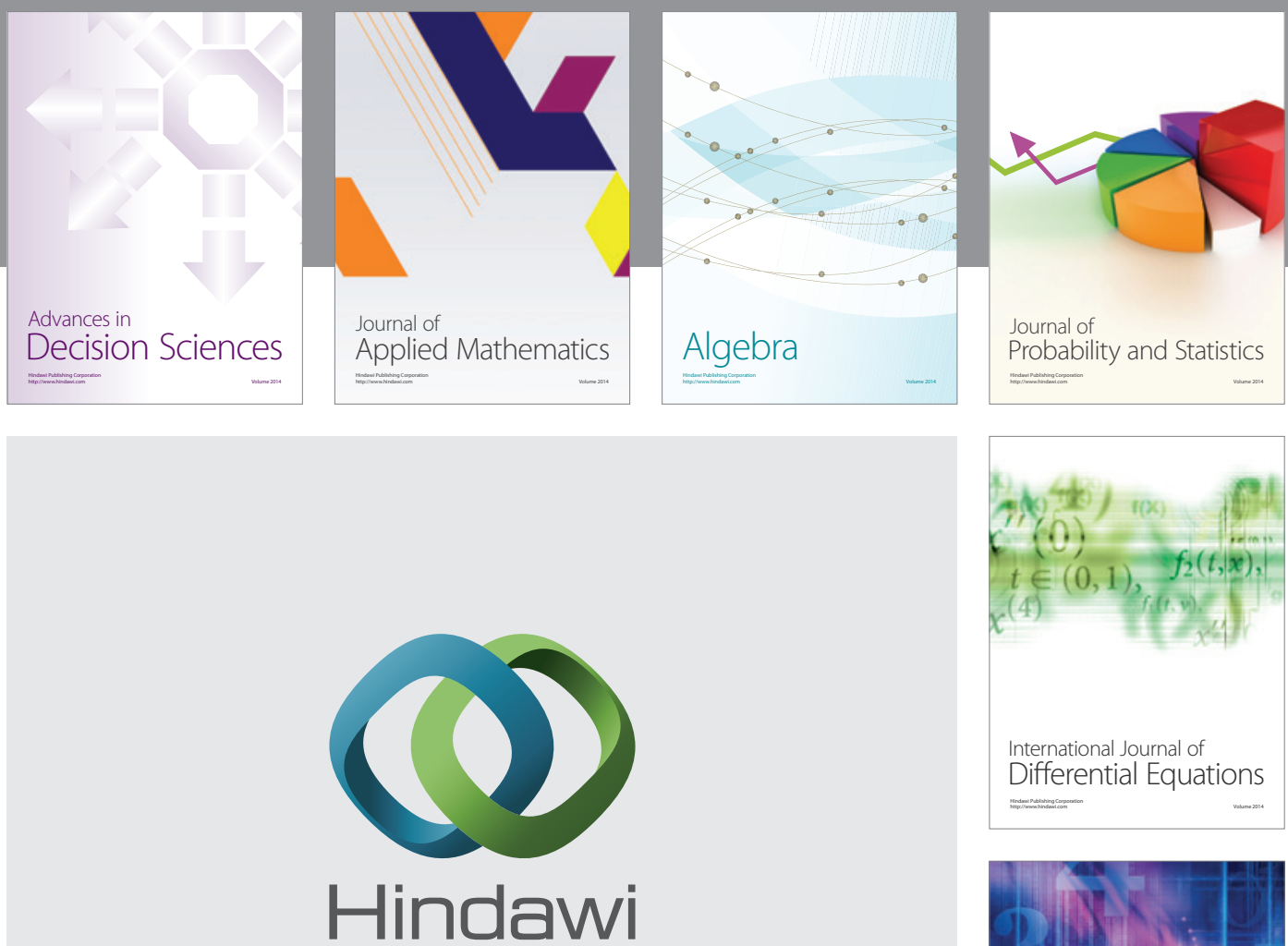

Submit your manuscripts at http://www.hindawi.com
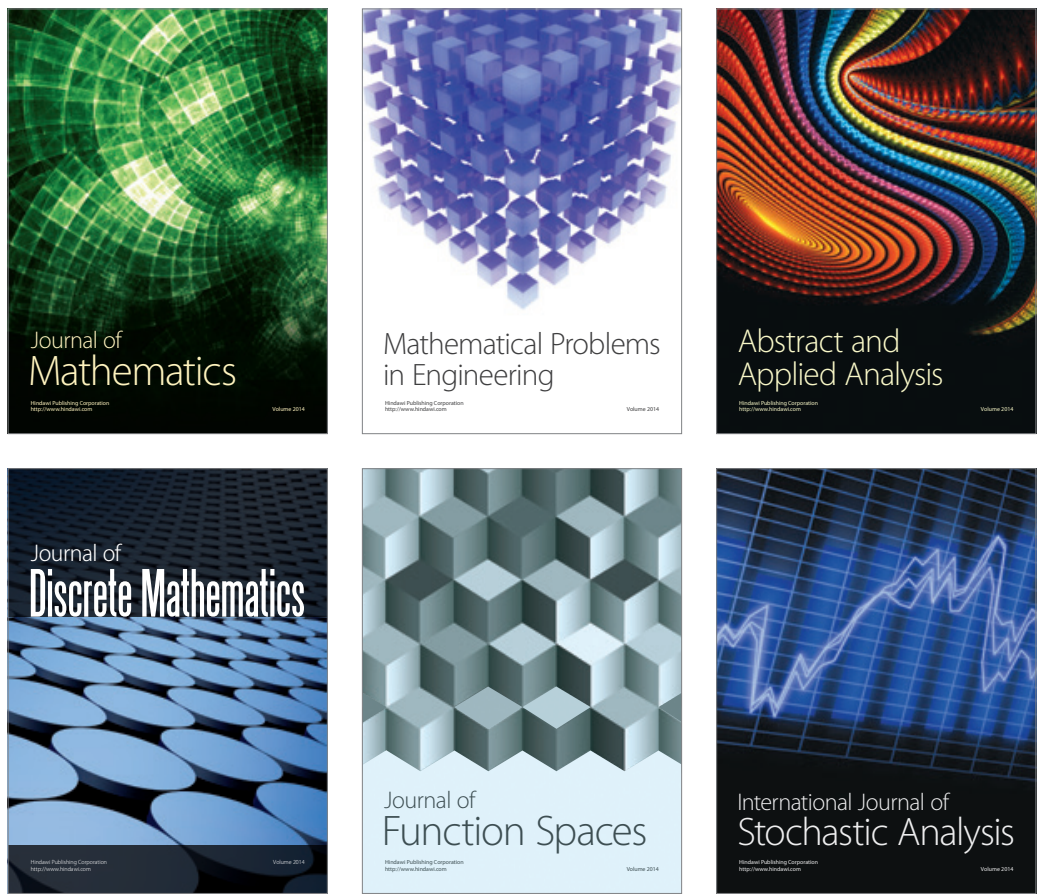

Journal of

Function Spaces

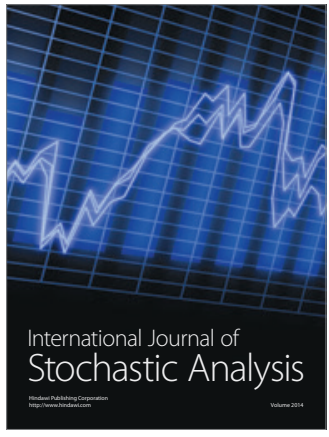

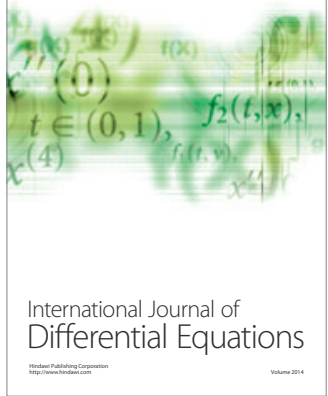
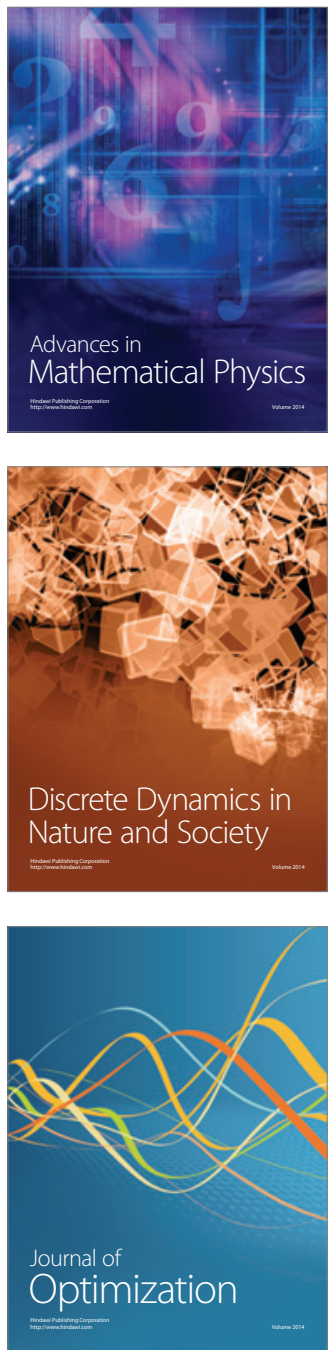